\title{
Parameter estimation of an extended inverse power Lomax distribution with Type I right censored data
}

\author{
Amal S. Hassan ${ }^{a}$, Said G. Nassr ${ }^{1, b}$ \\ ${ }^{a}$ Department of Mathematical Statistics, Cairo University, Egypt; \\ ${ }^{b}$ Department of Quantitative Methods, Sinai University, Egypt
}

\begin{abstract}
In this paper, we introduce an extended form of the inverse power Lomax model via Marshall-Olkin approach. We call it the Marshall-Olkin inverse power Lomax (MOIPL) distribution. The four- parameter MOIPL distribution is very flexible which contains some former and new models. Vital properties of the MOIPL distribution are affirmed. Maximum likelihood estimators and approximate confidence intervals are considered under Type I censored samples. Maximum likelihood estimates are evaluated according to simulation study. Bayesian estimators as well as Bayesian credible intervals under symmetric loss function are obtained via Markov chain Monte Carlo (MCMC) approach. Finally, the flexibility of the new model is analyzed by means of two real data sets. It is found that the MOIPL model provides closer fits than some other models based on the selected criteria.

Keywords: Inverse power Lomax distribution, Marshall-Olkin method, maximum likelihood, Bayesian estimation, Type I censored sample
\end{abstract}

\section{Introduction}

Lomax distribution is a heavy-tailed distribution usually employed in business, economics, and actuarial modeling. Lomax distribution has been used in various fields (Harris, 1968; Atkinson and Harrison, 1978; Holland et al., 2006; Hassan and Al-Ghamdi, 2009; Hassan et al., 2016). In the literature, extended and generalized forms of Lomax distribution have been considered by several authors. Examples include, Marshall-Olkin extended-Lomax distribution by Ghitany et al. (2007), exponentiated Lomax distribution by Abdul-Moniem and Abdel-Hameed (2012), beta Lomax, Kumaraswamy Lomax and McDonald Lomax by Lemonte and Cordeiro (2013), gamma-Lomax distribution by Cordeiro et al. (2013), Weibull Lomax distribution by Tahir et al. (2015), Gumbel-Lomax distribution by Tahir et al. (2016), power Lomax (PL) distribution by Rady et al. (2016), exponentiated Lomax geometric distribution by Hassan and Abd-Allah (2017), PL Poisson distribution by Hassan and Nassr (2018), exponentiated Weibull-Lomax distribution by Hassan and Abd-Allah (2018), inverse exponentiated Lomax distribution by Hassan and Mohamed (2019), Weibull inverse Lomax distribution by Hassan and Mohamed (2019), Type II Topp Leone power Lomax distribution by Al-Marzouk et al. (2020), truncated power Lomax distribution by Hassan et al. (2020) and half logistic Lomax distribution by Hassan et al. (2020) among others.

Recently, Hassan and Abd-Alla (2019) introduced the inverse PL (IPL) distribution which is a member of the inverted family of distributions. The IPL is very flexible in analyzing situations with

\footnotetext{
${ }^{1}$ Corresponding author: Department of Quantitative Methods, Faculty of Business Administration, Sinai University, AlArish, P.O. Box 45511, Egypt. E-mail: dr.saidstat@gmail.com
}

Published 31 March 2021 / journal homepage: http://csam.or.kr

(C) 2021 The Korean Statistical Society, and Korean International Statistical Society. All rights reserved. 
a realized non-monotonic failure rate. The IPL distribution with shape parameters $\alpha, \beta$ and scale parameter $\theta$ has the following probability density function (pdf)

$$
g(y ; \alpha, \beta, \theta)=\alpha \beta \theta^{\beta} y^{-(\alpha+1)}\left(\theta+y^{-\alpha}\right)^{-\beta-1} ; \quad y, \alpha, \beta, \theta>0 .
$$

The cumulative distribution function (cdf) related to (1.1) is defined by

$$
G(y ; \alpha, \beta, \theta)=\theta^{\beta}\left(\theta+y^{-\alpha}\right)^{-\beta} .
$$

On the other hand, Marshall and Olkin (1997) introduced a new family of survival functions which is obtained by adding a new parameter $\delta>0$ to an existing distribution. The new parameter will result in flexibility in the distribution. This family is called the Marshall-Olkin-G (MO-G) class. Let $\bar{G}(y)=1-G(y)$ is the survival function of a random variable $Y$, the cdf and pdf of the MO-G family are defined as follows:

$$
F_{\mathrm{MO}}(y)=\frac{G(y)}{[1-\bar{\delta}(\bar{G}(y))]},
$$

and

$$
f_{\mathrm{MO}}(y)=\frac{\delta g(y)}{[1-\bar{\delta}(\bar{G}(y))]^{2}},
$$

where, $\delta>0$ and $\bar{\delta}=1-\delta$ is known as a tilt parameter. The relationship between the hazard rate function (hrf) of the original distribution $h(y)$ and hrf of MO distribution is

$$
r_{\mathrm{MO}}(y ; \delta)=\frac{h(y)}{[1-\bar{\delta}(\bar{G}(y))]},
$$

which follows that $r_{\mathrm{MO}}(y: \delta) / h(y)$, is increasing in $y$ for $\delta \geq 1$ and decreasing for $0<\delta \leqslant 1$. In the sense that MO-G distribution gives more flexibility for modelling various types of real data in practice. Based on the above MO transformation several researchers have considered various extended distributions in the last few years. For instance, Marshall and Olkin (1997) developed the MO exponential and MO Weibull distributions, Rao et al. (2009) proposed the MO extended Lomax distribution, Krishna et al. (2013) proposed the MO Fréchet distribution, MO exponential Weibull distribution has been discussed by Pogány et al. (2015), MO power Lomax distribution has been introduced by Haq et al. (2020) and generalized MO inverse Lindley by Bantan et al. (2020) among others.

In many life test problems, the experimenter may not always obtain total information on failure times units for the sake of cost and time considerations. The observed data from life test experiments are referred to censored data. Life tests terminated at a pre-determined time, say $\tau$ are known as Type I censored (TIC) samples.

Two contributions motivated by the MO transformation and TIC samples are regarded. First, we use the basic motivations of the MOIPL distribution in practice are (i) it follows by considering a parallel system with $\mathrm{Z}$ independent components and supposes that a random variable $\mathrm{Z}$ has the geometric distribution with the probability mass function $P(Z=z)=\delta^{-1}\left(1-\delta^{-1}\right)^{z-1}, z=1,2, \ldots$ and $\delta>1$. Let $T_{1}, T_{2}, \ldots$ represent the lifetimes of each component and suppose that they have the IPL 
distribution defined in (1.1). Then a random variable $Y=\max \left(T_{1}, T_{2}, \ldots, T_{z}\right)$ represents the lifetime of the system. Therefore, the random variable $Y$ follows the MOIPL model, (ii) to obtain more flexible pdf with right skewed, uni-modal, reversed $J$ and symmetric shapes; (iii) It is interesting to observe that its hrf can be increasing, decreasing, bathtub or upside-down, and (iv) to provide significant improvement in data modeling. Second, we obtain the maximum likelihood (ML) and Bayesian estimators of the population parameters in case of the TIC scheme. Further, approximate confidence intervals (CIs) and Bayesian credible intervals (BCIs) are obtained. This paper is designed as follows. Section 2 defines the MOIPL distribution and provides its sub-models. Main properties of the MOIPL distribution are derived in Section 3. The ML estimators and CIs of the parameters as well as numerical issues are obtained in Section 4. Bayesian estimators are developed in Section 5. MCMC approach is employed in Section 6. The usefulness of the new model using two applications is explored in Section 7. The article ends with concluding remarks.

\section{Marshel-Olkin inverse power Lomax distribution}

In this section, an extended form of the IPL model is introduced. The new distribution is developed based on MO approach. The MOIPL distribution is formulated by inducing a tilt parameter in the IPL distribution. We now give the notion of the MOIPL distribution.

Definition 1. A random variable $Y$ is said to have MOIPL distribution if its pdf is of the form

$$
f(y ; v)=\alpha \beta \delta \theta^{\beta} y^{-(\alpha+1)}\left(\theta+y^{-\alpha}\right)^{-\beta-1}\left\{1-\bar{\delta}\left[1-\theta^{\beta}\left(\theta+y^{-\alpha}\right)^{-\beta}\right]\right\}^{-2} ; \quad y>0,
$$

where, $v(\alpha, \beta, \theta, \delta)$ is a set of parameters. The cdf of MOIPL distribution related to (2.1) is defined by

$$
F(y ; v)=\theta^{\beta}\left(\theta+y^{-\alpha}\right)^{-\beta}\left\{1-\bar{\delta}\left[1-\theta^{\beta}\left(\theta+y^{-\alpha}\right)^{-\beta}\right]\right\}^{-1} .
$$

A random variable $Y$ has MOIPL distribution will be denoted by $Y \sim \operatorname{MOIPL}(\alpha, \beta, \theta, \delta)$.

- For $\delta=1$, we obtain the IPL distribution.

- For $\alpha=1$, and $\theta=1 / \phi$, we obtain the MO inverse Pareto distribution (see M-Gharib et al., 2017).

- For $\delta=1$, and $\alpha=1$, we obtain inverse Lomax distribution.

- For $\delta=1$, and $Y=1 / X$, we obtain the PL distribution.

- For $\alpha=1$, and $Y=1 / X$, we obtain MO extended Lomax distribution.

- For $\delta=1, \alpha=1$, and $Y=1 / X$, we obtain Lomax distribution.

The survival function (sf) and hrf of MOIPL distribution are given, respectively, by

$$
\bar{F}(y ; v)=\delta\left[1-\theta^{\beta}\left(\theta+y^{-\alpha}\right)^{-\beta}\right]\left\{1-\bar{\delta}\left[1-\theta^{\beta}\left(\theta+y^{-\alpha}\right)^{-\beta}\right]\right\}^{-1},
$$

and

$$
h(y ; v)=\frac{\alpha \beta \theta^{\beta} y^{-(\alpha+1)}\left(\theta+y^{-\alpha}\right)^{-\beta-1}}{\left[1-\theta^{\beta}\left(\theta+y^{-\alpha}\right)^{-\beta}\right]\left\{1-\bar{\delta}\left[1-\theta^{\beta}\left(\theta+y^{-\alpha}\right)^{-\beta}\right]\right\}} .
$$



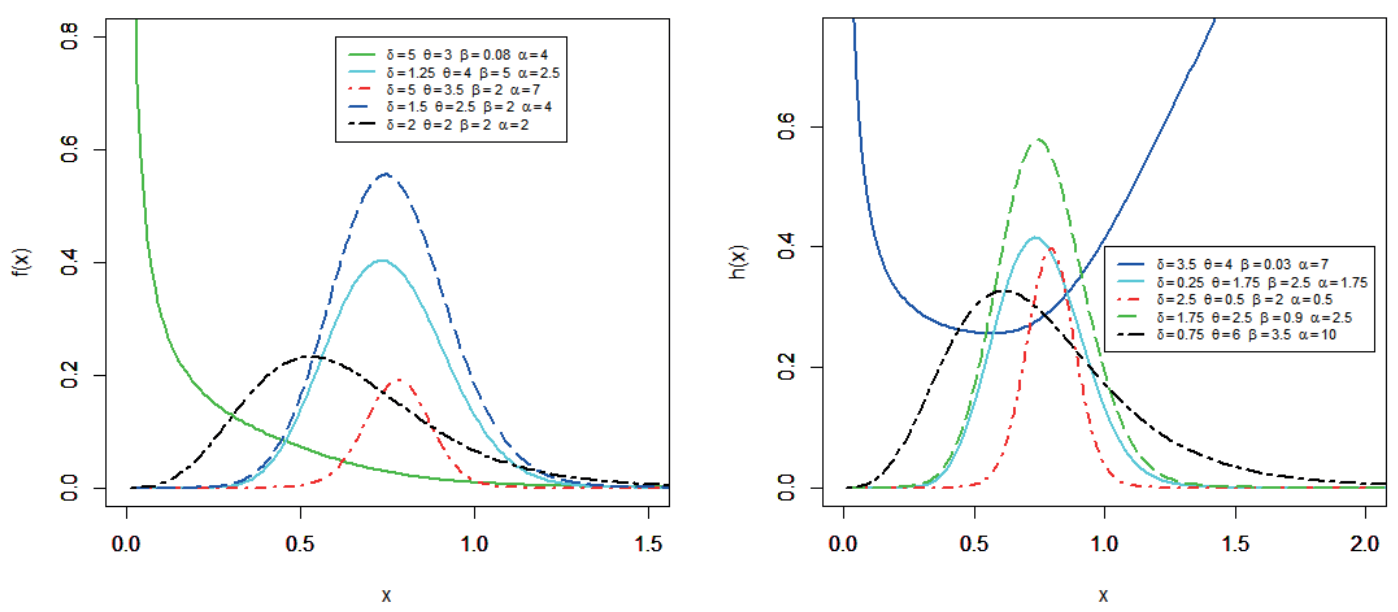

Figure 1: Different pdf and hrf plots of MOIPL distribution for some parameter values.

Figure 1 illustrates the shapes of the pdf and hrf of MOIPL distribution for some choices of parameters.

We notice that the pdf of MOIPL distribution can take different forms, so it is quite flexible. Also, it can be observed that the shapes of the hrf can be up-side down, bath-tub shaped at some selected values of parameters. Accordingly, it can be used effectively to analyze lifetime data sets.

\section{Structural properties}

Here, some statistical properties of MOIPL distribution including, quantile function, $s^{\text {th }}$ moment and $s^{\text {th }}$ negative moment, moments of the residual life, Rényi entropy and stochastic ordering are obtained.

\subsection{Moments of the MOIPL distribution}

Some important characteristics of a distribution like dispersion, skewness and kurtosis can be discussed through its moments. The $s^{\text {th }}$ moment of $Y$ is obtained by using pdf (2.1) as follows

$$
E\left(Y^{s}\right)=\int_{0}^{\infty} \alpha \beta \delta \theta^{\beta} y^{s-(\alpha+1)}\left(\theta+y^{-\alpha}\right)^{-\beta-1}\left\{1-\bar{\delta}\left[1-\theta^{\beta}\left(\theta+y^{-\alpha}\right)^{-\beta}\right]\right\}^{-2} d y .
$$

For $|t|<1, b>0$ we have the following series expansion

$$
(1-t)^{-b}=\Sigma_{k=0}^{\infty} \frac{\Gamma(k+b) t^{k}}{\Gamma(b) k !}
$$

Then employing (3.2) in (3.1), then

$$
E\left(Y^{s}\right)=\Sigma_{k=0}^{\infty}(k+1) \int_{0}^{\infty} \alpha \beta \delta \theta^{\beta} y^{s-(\alpha+1)}\left(\theta+y^{-\alpha}\right)^{-\beta-1}(\bar{\delta})^{k}\left[1-\theta^{\beta}\left(\theta+y^{-\alpha}\right)^{-\beta}\right]^{k} d y .
$$

Apply the binomial expansion in (3.3), then we have

$$
E\left(Y^{s}\right)=\Sigma_{k=0}^{\infty} \Sigma_{j=0}^{k}(-1)^{j}(k+1)\left(\begin{array}{c}
k \\
j
\end{array}\right) \int_{0}^{\infty} \alpha \beta \delta(\bar{\delta})^{k} \theta^{\beta+\beta j} y^{s-(\alpha+1)}\left(\theta+y^{-\alpha}\right)^{-(\beta+\beta j+1)} d y .
$$


Table 1: Some moments, skewness and kurtosis of $Y$ for selected parameters values

\begin{tabular}{cccccccc}
\hline \hline$\mu_{s}^{\prime}$ & (i) & (ii) & (iii) & (iv) & (v) & (vi) & (vii) \\
\hline$\mu_{1}^{\prime}$ & 0.816 & 1.211 & 1.083 & 1.037 & 1.306 & 1.134 & 0.843 \\
$\mu_{2}$ & 0.846 & 1.652 & 1.430 & 1.191 & 1.813 & 1.361 & 0.806 \\
$\mu_{3}$ & 1.128 & 2.678 & 2.356 & 1.513 & 2.710 & 1.748 & 0.867 \\
$\mu_{4}^{\prime}$ & 2.248 & 6.136 & 5.678 & 2.157 & 4.469 & 2.464 & 1.063 \\
$S$ & 1.874 & 2.868 & 1.931 & 0.925 & 1.709 & 1.804 & 0.916 \\
$K$ & 19.035 & 36.297 & 21.372 & 7.409 & 11.899 & 12.855 & 6.631 \\
\hline \hline & (i) $(\delta=0.5, \theta=0.5, \alpha=5, \beta=0.5),(\mathrm{ii})(\delta=0.5, \theta=0.5, \alpha=5, \beta=1.5),(\mathrm{iii})(\delta=1.5, \theta=0.5, \alpha=5, \beta=0.5),(\mathrm{iv})$ \\
$(\delta=1.5, \theta=0.5, \alpha=7, \beta=0.5),(\mathrm{v})(\delta=1.5, \theta=0.5, \alpha=7, \beta=1.5),(\mathrm{vi})(\delta=0.5, \theta=0.5, \alpha=7, \beta=1.5)$ and (vii) \\
$(\delta=0.5, \theta=0.5, \alpha=7, \beta=0.5)$.
\end{tabular}

After some simplification, the $s^{\text {th }}$ moment of MOIPL distribution is given by

$$
E\left(Y^{s}\right)=\Sigma_{k=0}^{\infty} A_{j} \theta^{\frac{-s}{\alpha}} \mathbf{B}\left(1-\frac{s}{\alpha}, \beta+\beta j+\frac{s}{\alpha}\right) \alpha>s,
$$

where, $A_{j}=\Sigma_{k=0}^{\infty}(k+1)\left(\begin{array}{l}k \\ j\end{array}\right) \beta \delta(\bar{\delta})^{k}$ and $\mathbf{B}(\cdot, \cdot)$ is the beta function.

Measures of skewness and kurtosis are calculated from the central moments using the well-known relationships. Further, the moment generating function is obtained as follows,

$$
M_{y}(t)=\Sigma_{k, s=0}^{\infty} \frac{\theta^{\frac{-s}{\alpha}} t^{s}}{s !} A_{j} \mathbf{B}\left(1-\frac{s}{\alpha}, \beta+\beta j+\frac{s}{\alpha}\right), \quad \alpha>s .
$$

The $s^{\text {th }}$ negative moment of MOIPL distribution (2.1) can be simply obtained as

$$
E\left(Y^{-s}\right)=\Sigma_{k=0}^{\infty} A_{j} \theta^{\frac{s}{\alpha}} \mathbf{B}\left(1+\frac{s}{\alpha}, \beta+\beta j-\frac{s}{\alpha}\right) .
$$

Additionally, the $s^{\text {th }}$ incomplete moment of $Y$ can be obtained from (2.1) as follows

$$
m_{s}(y)=\int_{0}^{y} \alpha \beta \delta \theta^{\beta} x^{s-(\alpha+1)}\left(\theta+x^{-\alpha}\right)^{-\beta-1}\left\{1-\bar{\delta}\left[1-\theta^{\beta}\left(\theta+x^{-\alpha}\right)^{-\beta}\right]\right\}^{-2} d x .
$$

Employing series expansion (3.2) and binomial expansion in (3.5), then we have

$$
m_{s}(y)=\Sigma_{k=0}^{\infty} A_{j} \theta^{\frac{-s}{\alpha}} \mathbf{B}\left(1-\frac{s}{\alpha}, \beta+\beta j+\frac{s}{\alpha}, \frac{\theta}{\theta+y^{-\alpha}}\right),
$$

where, $\mathbf{B}(\cdot, \cdot, t)$ is the incomplete beta function. The Bonferroni and Lorenz curves are an important application of $m_{1}(y)$, where $m_{1}(y)$ is the first incomplete moment. Another application involving $m_{1}(y)$ is related to the deviations from the mean and median.

Table 1 presents some empirical values of moments of order $1,2,3$, and 4 , the skewness $(S)$ and the kurtosis $(K)$ of $Y$ for some determined values of the parameters (i) $(\delta=0.5, \theta=0.5, \alpha=5, \beta=0.5)$, (ii) $(\delta=0.5, \theta=0.5, \alpha=5, \beta=1.5)$, (iii) $(\delta=1.5, \theta=0.5, \alpha=5, \beta=0.5)$, (iv) $(\delta=1.5, \theta=0.5, \alpha=$ 7, $\beta=0.5)$, (v) $(\delta=1.5, \theta=0.5, \alpha=7, \beta=1.5)$, (vi) $(\delta=0.5, \theta=0.5, \alpha=7, \beta=1.5)$ and (vii) $(\delta=0.5, \theta=0.5, \alpha=7, \beta=0.5)$.

\subsection{Conditional moments}

For lifetime models, it is of interest to obtain the conditional moments and the mean residual lifetime (MRL) function. The $n^{\text {th }}$ conditional moment is defined by

$$
\Xi_{n}(t)=\frac{1}{\bar{F}(t)} \int_{t}^{\infty}(y-t)^{n} f(y) d y .
$$


The $n^{\text {th }}$ moment of the residual life of MOIPL distribution is obtained by using pdf (2.1) and binomial expansion in (3.6) as follows

$$
\Xi_{n}(t)=\frac{\sum_{i=0}^{n}(-1)^{n-i}\left(\begin{array}{c}
n \\
i
\end{array}\right) t^{n-i}}{\bar{F}(t ; v)} \int_{t}^{\infty} \alpha \beta \delta \theta^{\beta} y^{n-(\alpha+1)}\left(\theta+y^{-\alpha}\right)^{-\beta-1}\left\{1-\bar{\delta}\left[1-\theta^{\beta}\left(\theta+y^{-\alpha}\right)^{-\beta}\right]\right\}^{-2} d y .
$$

Using series expansion (3.2) and binomial expansion in (3.7), then we have

$$
\Xi_{n}(t)=\frac{1}{\bar{F}(t ; v)} \Sigma_{k=0}^{\infty} \Sigma_{i=0}^{n}(-1)^{n-i}\left(\begin{array}{c}
n \\
i
\end{array}\right) t^{n-i} A_{j} \theta^{\frac{-n}{\alpha}} \mathbf{B}\left(1-\frac{n}{\alpha}, \beta+\beta j+\frac{n}{\alpha}, \frac{t^{-\alpha}}{\theta+t^{-\alpha}}\right),
$$

where $\mathbf{B}(\cdot, \cdot, t)$ is the incomplete beta function. For, $n=1$ in (3.8), we obtain the MRL of MOIPL model, which it has many applications in some fields.

Moreover, the reversed residual life (RRL) is defined as the conditional random variable $t-Y \mid Y \leqslant t$ which denotes the time elapsed from the failure of a component given that its life is less than or equal to $t$. The $n^{\text {th }}$ moment of the RRL is given by

$$
\omega_{n}(t)=\frac{1}{F(t)} \int_{0}^{t}(y-t)^{n} f(y) d y .
$$

The $n^{\text {th }}$ moment of the RRL of MOIPL distribution is obtained by binomial expansion twice time, and series expansion (3.2) in (3.9) as follows

$$
\omega_{n}(t)=\frac{1}{F(t ; v)} \sum_{k=0}^{\infty} \sum_{i=0}^{n}(-1)^{n-i}\left(\begin{array}{c}
n \\
i
\end{array}\right) t^{n-i} A_{j} \theta^{\frac{-n}{\alpha}} \mathbf{B}\left(1-\frac{n}{\alpha}, \beta+\beta j+\frac{n}{\alpha}, \frac{\theta}{\theta+t^{-\alpha}}\right), \quad \alpha>n .
$$

For, $n=1$ in (3.10), we get the mean of RRL which represents the waiting time elapsed since the failure of an item on condition that this failure had occurred. Also, it is known as the mean waiting time or the mean inactivity time.

\subsection{Rényi entropy}

The entropy affords great tool to evaluate the amount of information (or uncertainty) exists in a random observation relating to its parent distribution. A small value of entropy provides the smaller uncertainty in the data. The Rényi entropy of a random variable $Y$ is defined by

$$
\operatorname{Re}_{\lambda}(Y)=\frac{1}{1-\lambda} \log \int_{-\infty}^{\infty} f(y)^{\lambda} d y, \quad \lambda>0 \text { and } \lambda \neq 1 .
$$

The Rényi entropy of MOIPL distribution is obtained by using pdf (2.1), binomial expansion and expansion (3.2) as follows

$\operatorname{Re}_{\lambda}(Y)=\frac{1}{1-\lambda} \log \left[\Sigma_{k=0}^{\infty} \Sigma_{j=0}^{k} \frac{(-1)^{j} \Gamma(k+2 \lambda)}{\Gamma(2 \lambda) k !}\left(\begin{array}{c}k \\ j\end{array}\right)\left(\alpha \beta \delta \theta^{\beta}\right)^{\lambda}(\bar{\delta})^{k} \theta^{\beta j} \int_{0}^{\infty} y^{-\lambda(\alpha+1)}\left(\theta+y^{-\alpha}\right)^{-\lambda(\beta+1)-\beta j} d y\right]$.

So, after some manipulation, the Rényi entropy of MOIPL distribution is

$$
\operatorname{Re}_{\lambda}(Y)=\frac{1}{1-\lambda} \log \left[\sum_{k=0}^{\infty} D_{j} \theta^{\frac{(\lambda-1)}{\alpha}} \mathbf{B}\left(\frac{\lambda(\alpha+1)-1}{\alpha}, \lambda(\beta+1)+\beta j-\frac{\lambda(\alpha+1)-1}{\alpha}\right)\right],
$$


where,

$$
D_{j}=\Sigma_{j=0}^{k} \frac{(-1)^{j} \Gamma(k+2 \lambda)}{\Gamma(2 \lambda) k !}\left(\begin{array}{c}
k \\
j
\end{array}\right) \alpha^{\lambda-1}(\beta \delta)^{\lambda}(\bar{\delta})^{k} .
$$

\subsection{Stochastic ordering}

Let $X_{1}$ and $X_{2}$ are independent random variables with cdfs $F_{1}(x)$ and $F_{2}(x)$ respectively, then $X_{1}$ is said to be smaller than $X_{2}$ in the following ordering (Shaked and Shanthikumar, 1994); if the following holds;

- Stochastic order $\left(X_{1} \leqslant s r X_{2}\right)$, if $F_{1}(x) \geq F_{2}(x)$ for all $x$.

- Likelihood ratio order $\left(X_{1} \leqslant{ }_{l r} X_{2}\right)$, if $f_{1}(x) / f_{2}(x)$ is decreasing in $x$.

- Hazard rate order $\left(X_{1} \leqslant h r X_{2}\right)$, if $h_{1}(x) \geq h_{2}(x)$, for all $x$.

- Mean residual life order $\left(X_{1} \leqslant m r l X_{2}\right)$ if $m_{1}(x) \geq m_{2}(x)$, for all $x$.

Theorem 1. Let $X_{1} \sim \operatorname{MOIPL}\left(\alpha_{1}, \beta_{1}, \delta_{1}, \theta\right)$ and $X_{2} \sim \operatorname{MOIPL}\left(\alpha_{2}, \beta_{2}, \delta_{2}, \theta\right)$. If $\alpha_{1} \geq \alpha_{2}, \delta_{1} \geq \delta_{2}$,

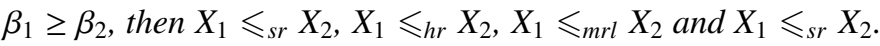

Proof: It is sufficient to show $f_{X_{1}}(x) / f_{X_{2}}(x)$ is a decreasing function of $x$; the likelihood ratio is

$$
\frac{f_{X_{1}}(x)}{f_{X_{2}}(x)}=\frac{\alpha_{1} \beta_{1} \delta_{1} \theta^{\beta_{1}} x^{-\left(\alpha_{1}+1\right)}\left(\theta+x^{-\alpha_{1}}\right)^{-\beta_{1}-1}\left\{1-\bar{\delta}_{1}\left[1-\theta^{\beta_{1}}\left(\theta+x^{-\alpha_{1}}\right)^{-\beta_{1}}\right]\right\}^{-2}}{\alpha_{2} \beta_{2} \delta_{2} \theta^{\beta_{2}} x^{-\left(\alpha_{2}+1\right)}\left(\theta+x^{-\alpha_{2}}\right)^{-\beta_{2}-1}\left\{1-\bar{\delta}_{2}\left[1-\theta^{\beta_{2}}\left(\theta+x^{-\alpha_{2}}\right)^{-\beta_{2}}\right]\right\}^{-2}} .
$$

Therefore,

$$
\begin{aligned}
\frac{d}{d x} \log \frac{f_{X_{1}}(x)}{f_{X_{2}}(x)}= & \frac{\left(\alpha_{2}-\alpha_{1}\right)}{x}-\frac{\alpha_{1}\left(\beta_{1}+1\right) x^{-\alpha_{1}-1}}{\theta+x^{-\alpha_{1}}}+\frac{\alpha_{2}\left(\beta_{2}+1\right) x^{-\alpha_{2}-1}}{\theta+x^{-\alpha_{2}}}+\frac{2 \bar{\delta}_{1} \theta^{\beta_{1}} \beta_{1} \alpha_{1}\left(\theta+x^{-\alpha_{1}}\right)^{-\beta_{1}-1} x^{-\alpha_{1}-1}}{\left\{1-\bar{\delta}_{1}\left[1-\theta^{\beta_{1}}\left(\theta+x^{-\alpha_{1}}\right)^{-\beta_{1}}\right]\right\}} \\
& -\frac{2 \bar{\delta}_{2} \theta^{\beta_{2}} \beta_{2} \alpha_{2}\left(\theta+x^{-\alpha_{2}}\right)^{-\beta_{2}-1} x^{-\alpha_{2}-1}}{\left\{1-\bar{\delta}_{2}\left[1-\theta^{\beta_{2}}\left(\theta+x^{-\alpha_{2}}\right)^{-\beta_{2}}\right]\right\}} .
\end{aligned}
$$

Now for $\alpha_{1} \geq \alpha_{2}, \delta_{1} \geq \delta_{2}$, and $\beta_{1} \geq \beta_{2}$, then $(d / d x) \log \left[f_{X_{1}}(x) / f_{X_{2}}(x)\right] \leqslant 0$, which implies that $X_{2}$ is stochastically greater than $X_{1}$ with respect to likelihood ratio order i.e., $X_{1} \leqslant{ }_{l r} X_{2}$ Similarly, we can conclude for $X_{1} \leqslant h r X_{2}, X_{1} \leqslant m l r X_{2}$, and $X_{1} \leqslant s r X_{2}$.

\subsection{Quantile function}

The quantile function of $Y$ has MOIPL distribution, for $q \in(0,1)$, is obtained by inverting (2.2) as follows

$$
\theta^{\beta}\left(\theta+y_{q}^{-\alpha}\right)^{-\beta}\left\{1-\bar{\delta}\left[1-\theta^{\beta}\left(\theta+y_{q}^{-\alpha}\right)^{-\beta}\right]\right\}^{-1}=q
$$

which gives;

$$
y_{q}=\theta^{\frac{-1}{\alpha}}\left[\left(\frac{q \delta}{1-q \bar{\delta}}\right)^{\frac{-1}{\beta}}-1\right]^{\frac{-1}{\alpha}}, \quad 0<q<1 .
$$


Table 2: Percentage points for some values of parameters

\begin{tabular}{ccccccc}
\hline \hline Percentage point & (a) & (b) & (c) & (d) & (e) & $(\mathrm{f})$ \\
\hline $25 \%$ & 0.0017 & 0.7521 & 1.5079 & 0.8293 & 0.7856 & 0.7898 \\
$50 \%$ & 0.0625 & 1.8517 & 2.8965 & 5.2486 & 1.2819 & 1.2025 \\
$75 \%$ & 1.2656 & 4.9295 & 5.8011 & 39.899 & 2.1581 & 1.7415 \\
$85 \%$ & 5.8000 & 8.9581 & 8.7729 & 135.895 & 2.9430 & 2.0524 \\
$95 \%$ & 81.450 & 28.9861 & 19.4827 & 1469.224 & 5.3540 & 2.4843 \\
\hline \hline$(\mathrm{a})(\delta=0.5, \theta=0.5, \alpha=0.5, \beta=0.5),(\mathrm{b})(\delta=0.5, \theta=0.5, \alpha=1, \beta=1.5),(\mathrm{c})(\delta=1.5, \theta=0.5, \alpha=1.5, \beta=1.5),(\mathrm{d})$ \\
$(\delta=1.5, \theta=1.5, \alpha=0.5, \beta=2),(\mathrm{e})(\delta=1.5, \theta=1.5, \alpha=2, \beta=1.5)$, and $(\mathrm{f})(\delta=0.8, \theta=1, \alpha=2, \beta=1.5)$.
\end{tabular}

Putting, $q=0.25,0.5$ and 0.75 in (3.11), we get the first quantile (25\%), median (50\%) and third quantile $(75 \%)$. If $U$ is a uniform variate on the unit interval $(0,1)$, then the random variable $Y=y_{q}$ at $q=U$ follows (2.2). By solving Equation (3.11) numerically, the percentage points $y_{q}$ are computed at some determined values of the parameters. (a) $(\delta=0.5, \theta=0.5, \alpha=0.5, \beta=0.5)$, (b) $(\delta=0.5, \theta=$ $0.5, \alpha=1, \beta=1.5),(\mathrm{c})(\delta=1.5, \theta=0.5, \alpha=1.5, \beta=1.5),(\mathrm{d})(\delta=1.5, \theta=1.5, \alpha=0.5, \beta=2),(\mathrm{e})$ $(\delta=1.5, \theta=1.5, \alpha=2, \beta=1.5)$, and (f) $(\delta=0.8, \theta=1, \alpha=2, \beta=1.5)$. These numerical values are provided in the Table 2 .

\section{ML estimation based on TIC samples}

Here, the ML estimators of the model parameters are determined via TIC scheme. Approximate CIs are obtained.

Let $Y=\left(Y_{1: n}<Y_{2: n}<\cdots<Y_{r: n}\right)$ is of TIC sample of size $r$ from a life test on $n$ items whose lifetimes have the MOIPL distribution with set of parameters $v=(\alpha, \theta, \beta, \delta)$. Based on TIC, the test is stopped at specified time $\tau$ before all $n$ items have failed. For simplicity, we write $y_{i}$ instead of $y_{i: n}$, hence the likelihood function in this situation is given by

$$
L(v \mid \underline{y})=\left(\alpha \beta \theta^{\beta}\right)^{r} \delta^{n}\left[\Pi_{i=1}^{r} y_{i}^{-(\alpha+1)} w_{i}^{-(\beta+1)}\left(1-\bar{\delta} q_{i}\right)^{-2}\right]\left[q^{*}\right]^{(n-r)}\left[1-\bar{\delta} q^{*}\right]^{-(n-r)},
$$

where; $w_{i}=\left(\theta+y_{i}^{-\alpha}\right), w^{*}=\left(\theta+\tau^{-\alpha}\right), q_{i}=\left(1-\theta^{\beta} w_{i}^{-\beta}\right)$, and $q^{*}=\left(1-\theta^{\beta}\left(w^{*}\right)^{-\beta}\right)$.

The log-likelihood function for the vector of parameters $v=(\alpha, \theta, \beta, \delta)$ is

$$
\begin{aligned}
\ln L(v \mid \underline{y}) \propto & r \ln \alpha+r \ln \beta+n \ln \delta+r \beta \ln \theta-(\alpha+1) \sum_{i=1}^{r} \ln y_{i}-(\beta+1) \sum_{i=1}^{r} \ln \left(w_{i}\right) \\
& -2 \Sigma_{i=1}^{r} \ln \left(1-\bar{\delta} q_{i}\right)+(n-r) \ln q^{*}-(n-r) \ln \left(1-\bar{\delta} q^{*}\right) .
\end{aligned}
$$

The partial derivatives with respect to the unknown parameters are given as follows:

$$
\begin{aligned}
\frac{\partial \ln L(v \mid \underline{y})}{\partial \alpha}= & \frac{r}{\alpha}-\Sigma_{i=1}^{r} \ln y_{i}+(\beta+1) \Sigma_{i=1}^{r} y_{i}^{-\alpha} \ln y_{i} w_{i}^{-1}-2 \beta \theta^{\beta} \bar{\delta} \Sigma_{i=1}^{r} y_{i}^{-\alpha} \ln y_{i} w_{i}^{-(\beta+1)}\left[1-\bar{\delta} q_{i}\right]^{-1} \\
& -(n-r) \beta \theta^{\beta} \tau^{\alpha} \ln \tau\left(w^{*}\right)^{-(\beta+1)}\left[\bar{\delta}\left(1-\bar{\delta} q^{*}\right)^{-1}-\left(q^{*}\right)^{-1}\right] \\
\frac{\partial \ln L(v \mid \underline{y})}{\partial \theta}= & \frac{r \beta}{\theta}-(\beta+1) \sum_{i=1}^{r} w_{i}^{-1}-2 \bar{\delta} \beta \theta^{\beta-1} \Sigma_{i=1}^{r} w_{i}^{-\beta}\left[1-\bar{\delta} q_{i}\right]^{-1}+2 \bar{\delta} \beta \theta^{\beta} \Sigma_{i=1}^{r} w_{i}^{-(\beta+1)}\left[1-\bar{\delta} q_{i}\right]^{-1} \\
& +(n-r) \beta \theta^{\beta-1}\left[\theta\left(w^{*}\right)^{-(\beta+1)}-\left(w^{*}\right)^{-\beta}\right]\left[\left(q^{*}\right)^{-1}+\bar{\delta}\left(1-\bar{\delta}\left(q^{*}\right)^{-1}\right)^{-1}\right],
\end{aligned}
$$




$$
\begin{aligned}
\frac{\partial \ln L(v \mid \underline{y})}{\partial \beta}= & \frac{r}{\beta}+r \ln \theta-\Sigma_{i=1}^{r} \ln \left(w_{i}\right)+2 \bar{\delta} \theta^{\beta} \Sigma_{i=1}^{r} w_{i}^{-\beta}\left[\ln \left(w_{i}\right)-\ln \theta\right]\left[1-\bar{\delta} q_{i}\right]^{-1} \\
& +(n-r) \theta^{\beta}\left(w^{*}\right)^{-\beta}\left[\ln \left(w^{*}\right)-\ln \theta\right]\left[\bar{\delta}\left\{1-\bar{\delta}\left(q^{*}\right)^{-1}\right\}^{-1}+\left\{q^{*}\right\}^{-1}\right], \\
\frac{\partial \ln L(v \mid \underline{y})}{\partial \delta}= & \frac{n}{\delta}-2 \Sigma_{i=1}^{r} q_{i}\left[1-\bar{\delta} q_{i}\right]^{-1}-(n-r) q^{*}\left[1-\bar{\delta} q^{*}\right]^{-1} .
\end{aligned}
$$

The ML estimators of the model parameters are the solution of non-linear Equations (4.2) to (4.5) after setting them equal to zero. These equations are very difficult to obtain, so iterative procedures are employed.

In case of interval estimation of the model parameters, we must obtain the $4 \times 4$ observed information matrix $I=I(v)$ (for $u, v=\alpha, \theta, \beta, \delta)$. Under standard regularity conditions, the multivariate normal $N_{4}\left(0, I^{-1}(\hat{v})\right)$ distribution is used to construct approximate CIs for the parameters. The approximate $100(1-\gamma) \%$ two sided CIs for $\alpha, \theta, \beta, \delta$ are respectively, given by:

$$
\hat{\alpha} \pm Z_{\frac{\gamma}{2}} \sigma_{\hat{\alpha}}, \quad \hat{\theta} \pm Z_{\frac{\gamma}{2}} \sigma_{\hat{\theta}}, \quad \hat{\beta} \pm Z_{\frac{\gamma}{2}} \sigma_{\hat{\beta}}, \quad \text { and } \quad \hat{\delta} \pm Z_{\frac{\gamma}{2}} \sigma_{\hat{\delta}}
$$

where, $Z_{\gamma / 2}$ is the $[100(1-\gamma) / 2]^{\text {th }}$ standard normal percentile and $\sigma(\cdot)$ is the standard deviation for the ML estimators.

\section{Bayesian estimation via TIC}

Here, the Bayesian estimators of the model parameters are obtained based on TIC scheme. The Bayesian estimator using squared error loss (SELF) function under the assumption of non-informative of the population parameters for MOIPL distribution is obtained. We consider the Bayesian estimation under the assumption that the random variables $(\alpha, \theta, \beta, \delta)$ independently distributed with the following uniform priors

$$
g(\alpha) \propto \alpha^{-1}, \quad g(\theta) \propto \theta^{-1}, \quad g(\beta) \propto \beta^{-1}, \quad \text { and } \quad g(\delta) \propto \delta^{-1} .
$$

Hence, the joint prior pdf of the parameters can be expressed by

$$
\pi^{*}(\alpha, \theta, \beta, \delta) \propto(\alpha \theta \beta \delta)^{-1} .
$$

Combining (4.1) and (5.1) to obtain the posterior density of $v=(\alpha \theta \beta \delta)$ given the data as follows

$$
\pi(v \mid y) \propto(\alpha \beta)^{r-1} \theta^{r \beta-1} \delta^{n-1}\left[\Pi_{i=1}^{r} y_{i}^{-(\alpha+1)} w_{i}^{-(\beta+1)}\left(1-\bar{\delta} q_{i}\right)^{-2}\right]\left[q^{*}\left(1-\bar{\delta} q^{*}\right)^{-1}\right]^{(n-r)} .
$$

Therefore, the Bayesian estimators of parameters, $v=(\alpha \theta \beta \delta)$ under SELF; denoted by $\tilde{u}_{(\mathrm{SELF})}(v)$ can be calculated through the following equations as follows

$$
\tilde{u}_{(\mathrm{SELF})}(v)=E(v \mid \underline{y})=\int_{0}^{\infty} \int_{0}^{\infty} \int_{0}^{\infty} \int_{0}^{\infty} v L(v \mid \underline{y}) \pi(v \mid \underline{y}) d \alpha d \theta d \beta d \delta .
$$

Generally, the ratio of four integrals given by Equations (5.2) and (5.3) cannot be obtained in a closed form. In this case, we use the MCMC technique to generate samples from the posterior distributions 
and then compute the Bayesian estimators of the individual parameters. The conditional posterior densities of $\alpha, \theta, \beta$, and $\delta$ are as follows

$$
\begin{aligned}
& \pi_{1}(\alpha \mid \underline{y}) \propto \alpha^{r-1}\left[\Pi_{i=1}^{r} y_{i}^{-(\alpha+1)} w_{i}^{-(\beta+1)}\left(1-\bar{\delta} q_{i}\right)^{-2}\right]\left[q^{*}\left(1-\bar{\delta} q^{*}\right)^{-1}\right]^{(n-r)}, \\
& \pi_{2}(\theta \mid \underline{y}) \propto \theta^{r \beta-1}\left[\Pi_{i=1}^{r} w_{i}^{-(\beta+1)}\left(1-\bar{\delta} q_{i}\right)^{-2}\right]\left[q^{*}\left(1-\bar{\delta} q^{*}\right)^{-1}\right]^{(n-r)}, \\
& \pi_{3}(\beta \mid \underline{y}) \propto \beta^{r-1} \theta^{r \beta-1}\left[\Pi_{i=1}^{r} w_{i}^{-(\beta+1)}\left(1-\bar{\delta} q_{i}\right)^{-2}\right]\left[q^{*}\left(1-\bar{\delta} q^{*}\right)^{-1}\right]^{(n-r)}, \\
& \pi_{4}(\delta \mid \underline{y}) \propto \delta^{n-1}\left[\Pi_{i=1}^{r}\left(1-\bar{\delta} q_{i}\right)^{-2}\right]\left[1-\bar{\delta} q^{*}\right]^{(n-r)} .
\end{aligned}
$$

Therefore, to generate from this distributions, we use the Metropolis-Hastings method. To run the Gibbs sampler algorithm, we start with the ML estimates (MLEs). We then draw samples from various full conditionals, in run, using the most recent values of all other conditioning variables unless some systematic pattern of convergence is achieved.

\section{Monte Carlo simulation}

In this section, a simulation study is carried out to evaluate the performance of the MLE. Measures like mean square errors (MSEs), standard errors (SEs), and average lengths (ALs) of the CIs are calculated. The simulation study is formed as follows:

Step 1: 10,000 random samples of size $n=30,40,50,100$, and 150 are generated from MOIPL distribution by using relation (3.11).

Step 2: Four sets of parameter values are chosen as; Case $\mathrm{I} \equiv(\alpha=0.75, \theta=0.5, \beta=1.5, \delta=0.25)$, Case II $\equiv(\alpha=0.5, \theta=0.25, \beta=2, \delta=0.25)$, Case III $\equiv(\alpha=1.25, \theta=1.5, \beta=0.75, \delta=$ $0.75)$, and Case IV $\equiv(\alpha=1.5, \theta=0.25, \beta=0.75, \delta=0.5)$. The censoring time is selected as $\tau=2$ and 5 .

Step 3: MLEs and Bayes estimates (BEs) of the parameters are obtained.

Step 4: MCMC technique (as M-H algorithm) is used to get the BEs of $\alpha, \theta, \beta$, and $\delta$ under SELF via 10,000 iterations.

Step 5: Compute MSEs, SEs and ALs with confidence level $\gamma=0.95$ of all estimates and the results are listed in Table 3 and Table 4 . We notice the following about the performance of estimates:

- For all cases, it is clear that MSEs, SEs, ALs, and MC error of BEs and MLEs decrease as sample size increases (see for example Figure 2 and Figure 3).

- BEs have smallest MSE compared to MLEs (Figure 2 and Figure 3).

- The MSEs of the MLEs for $\delta$ are smaller than the corresponding for the other parameters of all the cases except Case III. Also, the ALs of the MLEs of $\delta$ take the smallest values compared to the other parameters in almost all of the cases.

- The MSEs of the BEs for $\alpha$ are smaller than the MSEs of the corresponding for the other parameters for all cases except Case IV.

- The MSEs of MLEs decrease as $\tau$ increases. Also, Case I has the smallest MSEs and ALs compared to the other cases. 
Table 3: MSEs, SEs and ALs of the MLEs and BEs for MOIPL distribution at $\tau=2$

\begin{tabular}{|c|c|c|c|c|c|c|c|c|c|c|}
\hline \multirow{2}{*}{$n$} & \multirow{2}{*}{ Method } & \multirow{2}{*}{ Measure } & \multicolumn{4}{|c|}{ Case I } & \multicolumn{4}{|c|}{ Case II } \\
\hline & & & $\alpha=0.75$ & $\beta=1.5$ & $\delta=0.25$ & $\theta=0.5$ & $\alpha=0.5$ & $\beta=2$ & $\delta=0.25$ & $\theta=0.25$ \\
\hline \multirow{7}{*}{30} & \multirow{3}{*}{ ML } & MSE & 0.0877 & 0.3703 & 0.0810 & 0.9634 & 0.0700 & 0.6536 & 0.0533 & 0.5217 \\
\hline & & SE & 0.0298 & 0.0936 & 0.0498 & 0.0737 & 0.0304 & 0.1383 & $2.5077 *$ & 0.1176 \\
\hline & & $\mathrm{AL}$ & 0.1170 & 0.3670 & 0.1950 & 0.2890 & 0.1190 & 0.5420 & $9.8300 *$ & 0.4610 \\
\hline & \multirow{4}{*}{ Bayesian } & MSE & $1.7570^{*}$ & 0.0510 & 0.1940 & 0.0170 & 0.1490 & 0.3920 & 0.1570 & 0.2820 \\
\hline & & SE & $0.5770^{*}$ & $3.5580 *$ & 0.0100 & $0.1290^{*}$ & $4.4070^{*}$ & 0.0130 & $3.5020 *$ & 0.0140 \\
\hline & & $\mathrm{AL}$ & 0.0140 & 0.2766 & 0.0108 & 0.3776 & 0.0277 & 0.0039 & 0.1891 & 0.4286 \\
\hline & & MC error & $0.2030 *$ & $1.9210^{*}$ & $5.7420 *$ & 0.0490 & $2.3510^{*}$ & $7.0590 *$ & $1.8910 *$ & $7.7210^{*}$ \\
\hline \multirow{7}{*}{40} & \multirow{3}{*}{ ML } & MSE & 0.0802 & 0.3168 & 0.0390 & 0.8793 & 0.0606 & 0.6005 & 0.0530 & 0.4560 \\
\hline & & SE & 0.0237 & 0.0726 & 0.0284 & 0.0483 & 0.0243 & 0.1145 & $1.8346^{*}$ & 0.0944 \\
\hline & & $\mathrm{AL}$ & 0.0930 & 0.2850 & 0.1110 & 0.1890 & 0.0950 & 0.4490 & $7.1920 *$ & 0.3700 \\
\hline & \multirow{4}{*}{ Bayesian } & MSE & $1.2650^{*}$ & 0.0390 & 0.1930 & 0.0150 & 0.1180 & 0.2590 & 0.1420 & 0.1920 \\
\hline & & SE & $1.5950 *$ & $7.1010^{*}$ & $8.5350 *$ & $0.2880 *$ & $1.3360 *$ & $4.9280 *$ & $3.0200 *$ & $8.4050^{*}$ \\
\hline & & $\mathrm{AL}$ & 0.0230 & 0.0560 & 0.1430 & $2.1000 *$ & 0.0770 & 0.2160 & 0.0050 & 0.2430 \\
\hline & & MC error & $0.9720 *$ & $4.4670^{*}$ & $5.4050 *$ & $0.1590 *$ & $0.7810^{*}$ & $3.0580 *$ & $1.8550 *$ & $5.3170^{*}$ \\
\hline \multirow{7}{*}{50} & \multirow{3}{*}{ ML } & MSE & 0.0750 & 0.2739 & 0.0240 & 0.8146 & 0.0552 & 0.5588 & 0.0528 & 0.3862 \\
\hline & & SE & 0.0197 & 0.0603 & 0.0177 & 0.0430 & 0.0187 & 0.0966 & $1.3310 *$ & 0.0772 \\
\hline & & $\mathrm{AL}$ & 0.0770 & 0.2370 & 0.0700 & 0.1690 & 0.0730 & 0.3790 & $5.2180 *$ & 0.3030 \\
\hline & \multirow{4}{*}{ Bayesian } & MSE & $0.4870^{*}$ & 0.0230 & 0.1930 & 0.0120 & 0.1060 & 0.2190 & 0.1200 & 0.1860 \\
\hline & & SE & $0.6430 *$ & $3.6420 *$ & $7.2520 *$ & $0.1970^{*}$ & $2.6740^{*}$ & $4.1210 *$ & $2.4880 *$ & $6.4810^{*}$ \\
\hline & & $\mathrm{AL}$ & 0.0080 & 0.0430 & 0.1410 & $1.8000^{*}$ & 0.0520 & 0.0610 & 0.0050 & 0.1620 \\
\hline & & MC error & $0.4110^{*}$ & $2.5430 *$ & $5.1150 *$ & $0.1120^{*}$ & $1.8770 *$ & $2.8550 *$ & $1.7400 *$ & $4.5750^{*}$ \\
\hline \multirow{7}{*}{100} & \multirow{3}{*}{ ML } & MSE & 0.0616 & 0.2537 & 0.0138 & 0.7667 & 0.0526 & 0.5371 & 0.0526 & 0.3626 \\
\hline & & SE & 0.0123 & 0.0383 & $5.7459 *$ & 0.0290 & 0.0118 & 0.0689 & $0.7611 *$ & 0.0536 \\
\hline & & $\mathrm{AL}$ & 0.0480 & 0.1500 & 0.0230 & 0.1140 & 0.0460 & 0.2700 & $2.9830 *$ & 0.2100 \\
\hline & \multirow{4}{*}{ Bayesian } & MSE & $0.2310^{*}$ & 0.0160 & 0.1910 & 0.0100 & 0.0960 & 0.2030 & 0.1140 & 0.1690 \\
\hline & & SE & $0.7360 *$ & $2.3470 *$ & 5.3950 & $0.1140 *$ & $1.9920 *$ & $3.3300 *$ & $1.7230 *$ & $4.3950^{*}$ \\
\hline & & $\mathrm{AL}$ & 0.0050 & 0.0240 & 0.1170 & $1.5000^{*}$ & 0.0450 & 0.0070 & 0.0040 & 0.1020 \\
\hline & & MC error & $0.6990 *$ & $2.3020 *$ & $5.3980 *$ & $0.0780 *$ & $1.9720^{*}$ & $3.3020 *$ & $1.7010 *$ & $4.3940 *$ \\
\hline \multirow{7}{*}{150} & \multirow{3}{*}{ ML } & MSE & 0.0585 & 0.1635 & 0.0114 & 0.6611 & 0.0504 & 0.5164 & 0.0525 & 0.3572 \\
\hline & & SE & $8.5658^{*}$ & 0.0254 & $4.2179 *$ & 0.0116 & $9.6159 *$ & 0.0522 & $0.6094 *$ & 0.0433 \\
\hline & & $\mathrm{AL}$ & 0.0340 & 0.1000 & 0.0170 & 0.0450 & 0.0380 & 0.2040 & $2.3890 *$ & 0.1700 \\
\hline & \multirow{4}{*}{ Bayesian } & MSE & $0.0090^{*}$ & 0.0150 & 0.1890 & $8.1920^{*}$ & 0.0730 & 0.1690 & 0.1010 & 0.1530 \\
\hline & & SE & $0.2470^{*}$ & $1.5730 *$ & $4.3470 *$ & $0.1060 *$ & $2.9030 *$ & $4.7660 *$ & $1.4790 *$ & $5.5580 *$ \\
\hline & & $\mathrm{AL}$ & 0.0050 & 0.0050 & 0.0270 & $1.3000 *$ & 0.0220 & 0.0060 & 0.0040 & 0.0660 \\
\hline & & MC error & $0.2510^{*}$ & $1.9070 *$ & $5.3240 *$ & $0.0090^{*}$ & $3.5560^{*}$ & $5.8360 *$ & $1.7840 *$ & $6.8270^{*}$ \\
\hline
\end{tabular}

* Indicate that the value multiply $10^{-3}$

- The MSEs of Bayes estimates decrease as $\tau$ increases. Also, the SEs of MLEs decrease as $\tau$ increases.

- The SEs of BEs for $(\alpha, \beta)$ based on Case IV, are smaller than the corresponding SEs for other parameters in most cases.

- The SEs of MLEs for $\delta$ are smaller than the SEs of the corresponding other parameters for all the cases. Also, the SEs of BEs for $\alpha$ are smaller than the corresponding SEs for other parameters in most cases.

- The SEs of the MLEs in Case I get the smallest values compared to the other cases.

- The SEs of MLE for $\delta$ are smaller than the corresponding for the other estimates for all cases.

- In Case I, the MLEs and BEs have good statistical properties than the corresponding cases of parameters in almost all of the situations (Figure 5). But in the Case IV the BEs for parameter $\alpha$ have smallest properties than other cases of parameters (Figures 4). 
Table 4: MSEs, SEs and ALs of the MLEs and BEs for MOIPL distribution at $\tau=5$

\begin{tabular}{|c|c|c|c|c|c|c|c|c|c|c|}
\hline \multirow{2}{*}{$n$} & \multirow{2}{*}{ Method } & \multirow{2}{*}{ Measure } & \multicolumn{4}{|c|}{ Case III } & \multicolumn{4}{|c|}{ Case IV } \\
\hline & & & $\alpha=1.25$ & $\beta=0.75$ & $\delta=0.75$ & $\theta=1.5$ & $\alpha=1.5$ & $\beta=0.75$ & $\delta=0.5$ & $\theta=0.25$ \\
\hline \multirow{7}{*}{30} & \multirow{3}{*}{ ML } & MSE & 0.1600 & 1.1383 & 0.5171 & 0.9427 & 0.6949 & 0.8598 & 0.2273 & 0.3375 \\
\hline & & SE & 0.0719 & 0.1178 & 0.0946 & 0.1435 & 0.0468 & 0.1012 & 0.0592 & 0.0889 \\
\hline & & $\mathrm{AL}$ & 0.2820 & 0.4620 & 0.3710 & 0.5620 & 0.1830 & 0.3970 & 0.2320 & 0.3490 \\
\hline & \multirow{4}{*}{ Bayesian } & MSE & 0.0238 & 0.0959 & 0.0111 & 0.5478 & 0.2561 & $2.4120^{*}$ & 0.1492 & 0.2754 \\
\hline & & SE & $1.3551 *$ & 0.0177 & 0.0100 & 0.0239 & $1.9960 *$ & $3.6330 *$ & 0.0110 & 0.0220 \\
\hline & & $\mathrm{AL}$ & 0.0140 & 0.2766 & 0.0108 & 0.3776 & 0.0277 & 0.0039 & 0.1891 & 0.4286 \\
\hline & & MC error & $0.4835^{*}$ & $9.7190 *$ & $5.2840 *$ & 0.0131 & $1.0620 *$ & $1.9660 *$ & $5.9710^{*}$ & 0.0192 \\
\hline \multirow{7}{*}{40} & \multirow{3}{*}{ ML } & MSE & 0.1351 & 1.1265 & 0.3658 & 0.9420 & 0.6878 & 0.7682 & 0.2209 & 0.2844 \\
\hline & & SE & 0.0557 & 0.0977 & 0.0427 & 0.1197 & 0.0348 & 0.0746 & 0.0484 & 0.0714 \\
\hline & & $\mathrm{AL}$ & 0.2180 & 0.3830 & 0.1680 & 0.4690 & 0.1360 & 0.2930 & 0.1900 & 0.2800 \\
\hline & \multirow{4}{*}{ Bayesian } & MSE & 0.0177 & 0.0806 & 0.0103 & 0.4951 & 0.1624 & $2.0475^{*}$ & 0.0845 & 0.2363 \\
\hline & & SE & $0.8579 *$ & $3.6710 *$ & $6.6790 *$ & 0.0210 & $0.4767^{*}$ & $3.2220 *$ & $5.9130 *$ & 0.0230 \\
\hline & & $\mathrm{AL}$ & 0.0070 & 0.0762 & 0.0056 & 0.3698 & 0.0080 & 0.0034 & 0.1029 & 0.4280 \\
\hline & & MC error & $0.3890 *$ & $2.2920 *$ & $4.0510 *$ & 0.0134 & $0.2135^{*}$ & $2.0040 *$ & $3.7340 *$ & 0.0148 \\
\hline \multirow{7}{*}{50} & \multirow{3}{*}{ ML } & MSE & 0.1127 & 1.0923 & 0.3404 & 0.8060 & 0.6844 & 0.7618 & 0.1492 & 0.2515 \\
\hline & & SE & 0.0438 & 0.0788 & 0.0374 & 0.0877 & 0.0310 & 0.0739 & 0.0208 & 0.0567 \\
\hline & & $\overline{\mathrm{AL}}$ & 0.1720 & 0.3090 & 0.1470 & 0.3440 & 0.1210 & 0.2900 & 0.0810 & 0.2220 \\
\hline & \multirow{4}{*}{ Bayesian } & MSE & 0.0171 & 0.0801 & 0.0101 & 0.4933 & 0.0408 & $1.7776^{*}$ & 0.0802 & 0.1700 \\
\hline & & SE & $0.7600^{*}$ & $1.3130 *$ & $5.9740 *$ & 0.0180 & $0.4560 *$ & $2.2660 *$ & $3.5950 *$ & 0.0150 \\
\hline & & $\mathrm{AL}$ & 0.0064 & 0.0110 & 0.0050 & 0.3692 & 0.0075 & 0.0030 & 0.1019 & 0.3830 \\
\hline & & MC error & $0.3113^{*}$ & $0.8149 *$ & $3.9960 *$ & 0.0127 & $0.1966^{*}$ & $1.5930 *$ & $2.5300 *$ & 0.0106 \\
\hline \multirow{7}{*}{100} & \multirow{3}{*}{ ML } & MSE & 0.0860 & 1.0869 & 0.3271 & 0.7856 & 0.6761 & 0.7409 & 0.1466 & 0.2477 \\
\hline & & SE & 0.0238 & 0.0520 & 0.0218 & 0.0671 & 0.0212 & 0.0491 & 0.0156 & 0.0409 \\
\hline & & $\mathrm{AL}$ & 0.0930 & 0.2040 & 0.0850 & 0.2630 & 0.0830 & 0.1920 & 0.0610 & 0.1600 \\
\hline & \multirow{4}{*}{ Bayesian } & MSE & 0.0156 & 0.0653 & 0.0096 & 0.4731 & 0.0118 & $0.4301^{*}$ & 0.0764 & 0.1599 \\
\hline & & SE & $0.6142 *$ & $1.3110 *$ & $3.9655^{*}$ & 0.0120 & $0.2329^{*}$ & $1.1590 *$ & $1.4430 *$ & 0.0120 \\
\hline & & $\mathrm{AL}$ & 0.0055 & 0.0090 & 0.0044 & 0.3594 & 0.0048 & $0.4301 *$ & 0.0764 & 0.1599 \\
\hline & & MC error & $0.3088^{*}$ & $0.2649 *$ & $3.7880 *$ & 0.0116 & $0.9897 *$ & $1.5911 *$ & $1.6020 *$ & 0.0090 \\
\hline \multirow{7}{*}{150} & \multirow{3}{*}{ ML } & MSE & 0.0798 & 1.0422 & 0.3167 & 0.5215 & 0.6707 & 0.7156 & 0.1383 & 0.2442 \\
\hline & & SE & 0.0184 & 0.0402 & 0.0173 & 0.0574 & 0.0171 & 0.0381 & $9.3703^{*}$ & 0.0314 \\
\hline & & $\mathrm{AL}$ & 0.0720 & 0.1570 & 0.0680 & 0.2040 & 0.0670 & 0.1490 & 0.0370 & 0.1230 \\
\hline & \multirow{4}{*}{ Bayesian } & MSE & 0.0130 & 0.0458 & $9.0341 *$ & 0.4448 & $3.0079^{*}$ & $0.3761 *$ & $0.0018^{*}$ & 0.1094 \\
\hline & & SE & $0.5257^{*}$ & $1.0570 *$ & $3.2910 *$ & $6.9400 *$ & $0.2081^{*}$ & $1.1344^{*}$ & $0.0966^{*}$ & $5.9850 *$ \\
\hline & & $\mathrm{AL}$ & 0.0032 & 0.0074 & $2.6400 *$ & $0.0556^{*}$ & 0.0014 & $0.0736^{*}$ & 0.0014 & 0.1267 \\
\hline & & MC error & $0.3607 *$ & $0.7434 *$ & $4.9270 *$ & $8.0370 *$ & $0.4623^{*}$ & $0.1880 *$ & $0.0540 *$ & 0.0073 \\
\hline
\end{tabular}

* Indicate that the value multiply $10^{-3}$

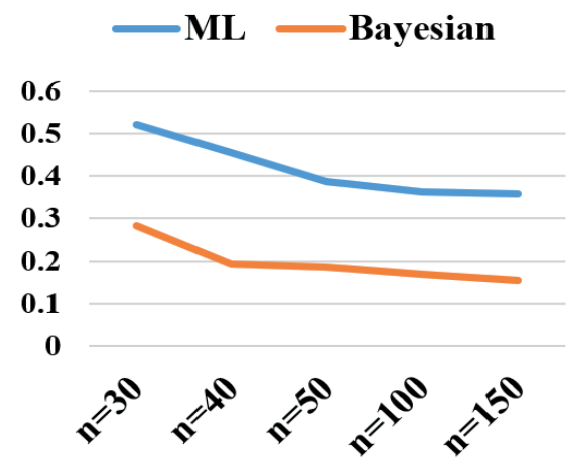

Figure 2: MSEs of $\hat{\theta}$ for Case II at $\tau=2$.

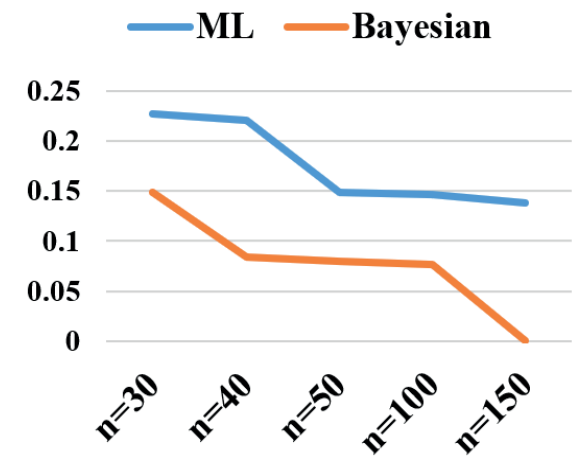

Figure 3: MSEs of $\hat{\delta}$ for Case $I V$ at $\tau=5$. 


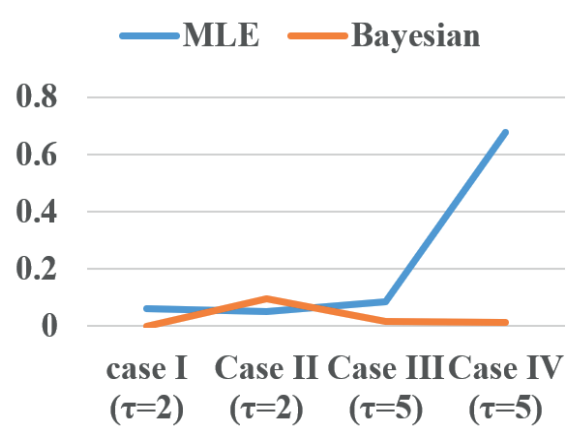

Figure 4: MSEs of $\hat{\alpha}$ for all Cases at $n=150$.

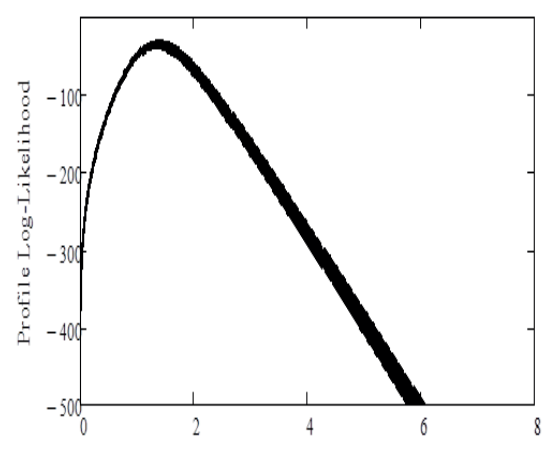

$\beta$

Figure 6: Profile log-likelihood of $\beta$.

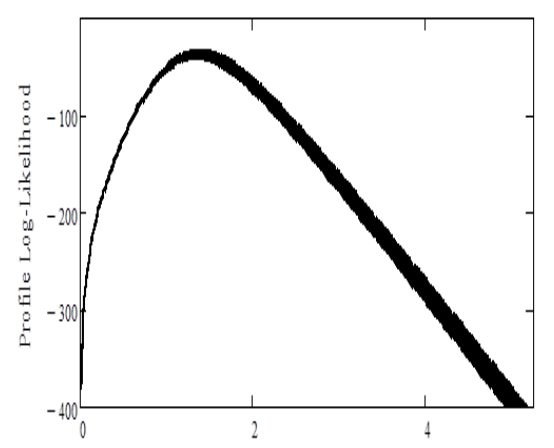

Figure 8: Profile log-likelihood of $\alpha$.

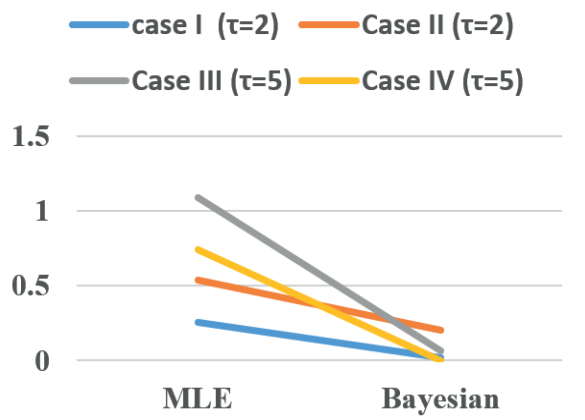

Figure 5: MSEs of $\hat{\beta}$ for all Cases at $n=100$.

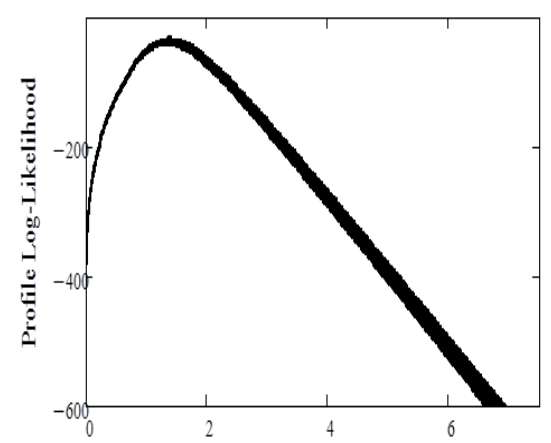

Figure 7: Profile log-likelihood of $\theta$.

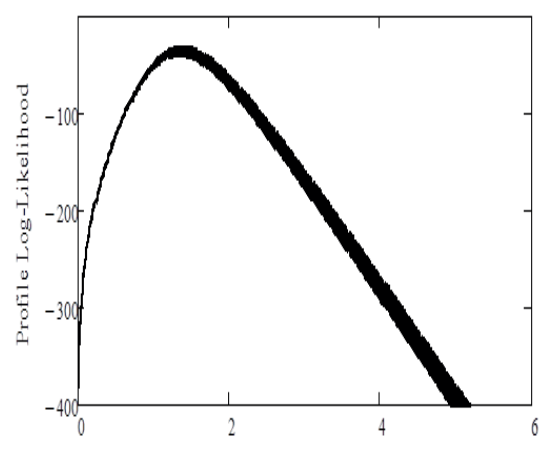

Figure 9: Profile log-likelihood of $\delta$.

- Figures 6-9 plots the profile log-likelihood function of $(\alpha, \theta, \beta, \delta)$. These plots are unimodal functions.

- The history plots of MCMC estimates for $\alpha, \theta, \beta$, and $\delta$ using MCMC sampler performance are represented in Figure 10 and Figure 11. The plots of chains for all parameters look like a horizontal band with no long upward or downward trends which are indicators to convergence. 

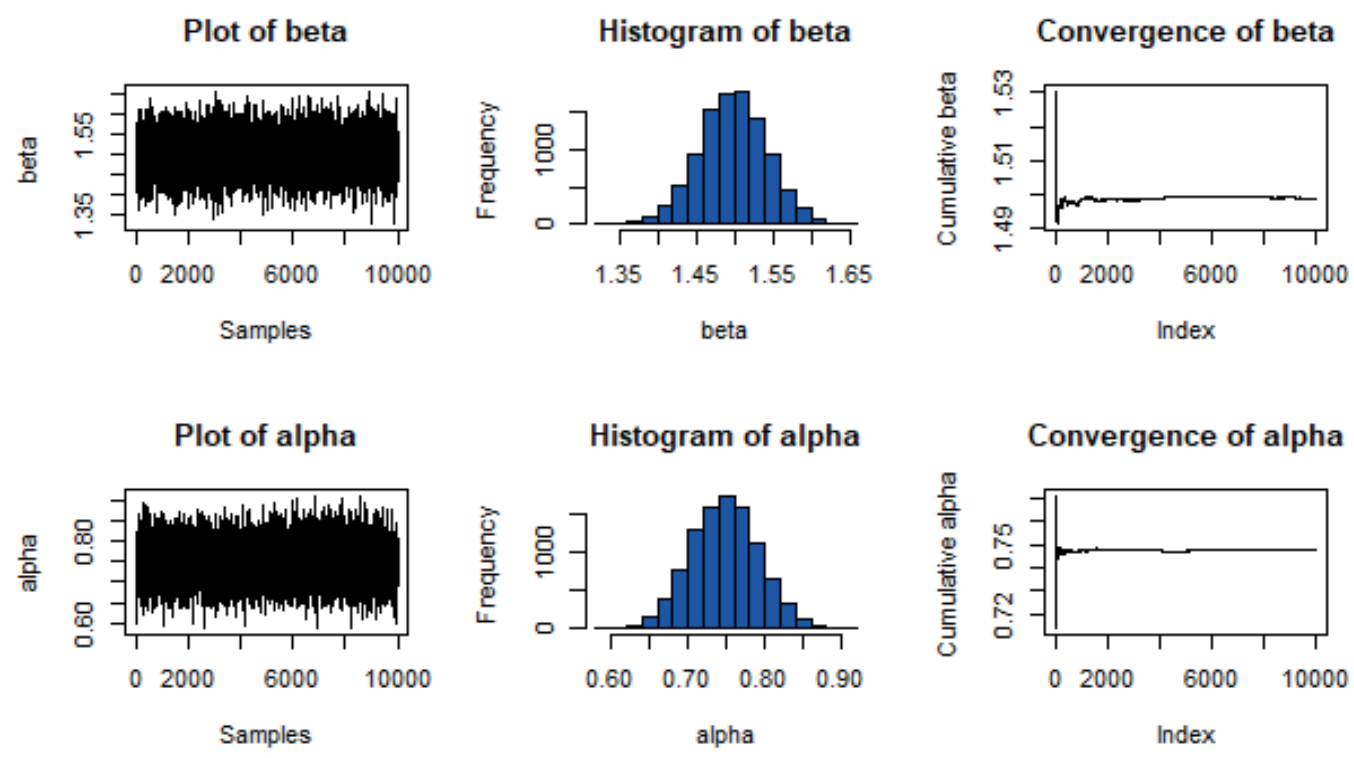

Figure 10: Convergence plots of MCMC estimates for $\alpha=0.75, \beta=1.5$ at $n=50$.

Plot of theta

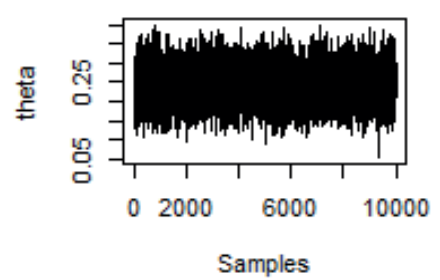

Plot of delta

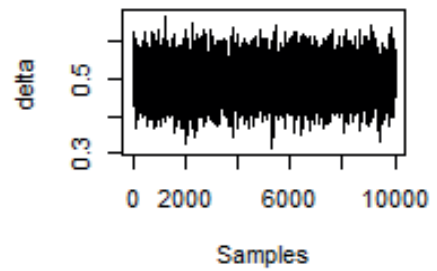

Histogram of theta

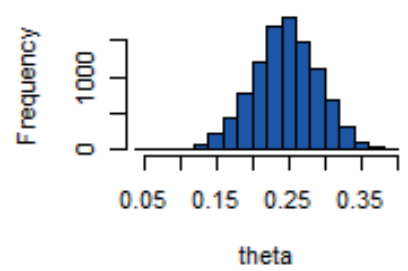

Histogram of delta

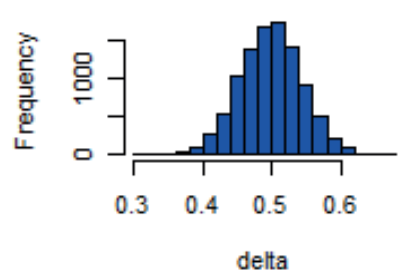

Convergence of theta

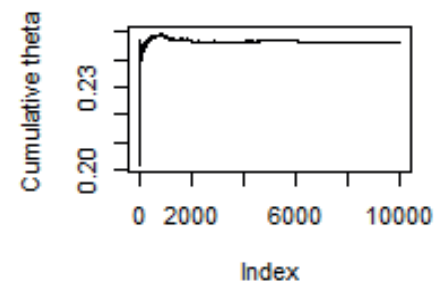

Convergence of delta

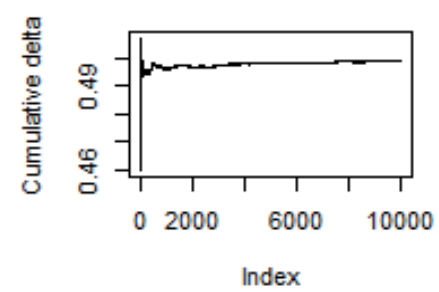

Figure 11: Convergence plots of MCMC estimates for $\delta=0.5, \theta=0.25$ at $n=100$.

\section{Real data modelling}

In this section, two real data sets are used to compare MOIPL model with IPL distribution Kumaraswamy Weibull Lomax (KWL) distribution, PL distribution, inverse exponentiated Lomax (IEL) distribution, Weibull inverse Lomax (WIL) distribution and exponentiated Lomax (EL). The two data sets are used 
Table 5: The MLEs, SEs and goodness-of-fit measures of the models for data set I

\begin{tabular}{|c|c|c|c|c|c|c|}
\hline Model & MLE & $\overline{\mathrm{SE}}$ & $P$-value & K-S & $W^{*}$ & $A^{*}$ \\
\hline \multirow{4}{*}{ MOIPL } & $\hat{\alpha}=3.280$ & 0.378 & \multirow{4}{*}{0.76486} & \multirow{4}{*}{0.23514} & \multirow{4}{*}{0.06152} & \multirow{4}{*}{0.34677} \\
\hline & $\hat{\beta}=0.291$ & 0.417 & & & & \\
\hline & $\hat{\delta}=46.403$ & 128.094 & & & & \\
\hline & $\hat{\theta}=3.230$ & 5.989 & & & & \\
\hline \multirow{4}{*}{ KWL } & $\hat{\alpha}=2.167$ & 8.435 & \multirow{4}{*}{0.63502} & \multirow{4}{*}{0.36498} & \multirow{4}{*}{0.08629} & \multirow{4}{*}{0.53444} \\
\hline & $\hat{\beta}=4.586$ & 12.963 & & & & \\
\hline & $\hat{\lambda}=3.025$ & 0.937 & & & & \\
\hline & $\hat{\theta}=6.410$ & 22.784 & & & & \\
\hline \multirow{4}{*}{ WIL } & $\hat{\lambda}=6.520$ & 13.090 & \multirow{4}{*}{0.64673} & \multirow{4}{*}{0.35327} & \multirow{4}{*}{0.08310} & \multirow{4}{*}{0.51486} \\
\hline & $\hat{a}=18.962$ & 14.618 & & & & \\
\hline & $\hat{b}=0.455$ & 0.927 & & & & \\
\hline & $\hat{\beta}=3.209$ & 2.746 & & & & \\
\hline \multirow{3}{*}{ IPL } & $\hat{\alpha}=0.690$ & 0.294 & \multirow{3}{*}{0.73882} & \multirow{3}{*}{0.26118} & \multirow{3}{*}{0.08981} & \multirow{3}{*}{0.62754} \\
\hline & $\hat{\lambda}=0.134$ & 0.145 & & & & \\
\hline & $\hat{\beta}=3.464$ & 0.725 & & & & \\
\hline \multirow{3}{*}{ EL } & $\hat{\lambda}=0.015$ & 0.043 & \multirow{3}{*}{0.55107} & \multirow{3}{*}{0.44893} & \multirow{3}{*}{0.08033} & \multirow{3}{*}{0.52282} \\
\hline & $\hat{\alpha}=3.716$ & 0.789 & & & & \\
\hline & $\hat{\theta}=78.322$ & 221.151 & & & & \\
\hline \multirow{3}{*}{ IEL } & $\hat{\alpha}=3.002$ & 0.955 & \multirow{3}{*}{0.62910} & \multirow{3}{*}{0.37090} & \multirow{3}{*}{0.08684} & \multirow{3}{*}{0.53680} \\
\hline & $\hat{\theta}=14.970$ & 11.248 & & & & \\
\hline & $\hat{\lambda}=2.828$ & 2.436 & & & & \\
\hline \multirow{3}{*}{ PL } & $\hat{\lambda}=6.1970$ & 9.837 & \multirow{3}{*}{0.68041} & \multirow{3}{*}{0.31959} & & \\
\hline & $\hat{\alpha}=1.780$ & 2.988 & & & 0.07169 & 0.44463 \\
\hline & $\hat{\beta}=2.573$ & 1.255 & & & & \\
\hline
\end{tabular}

to investigate the usefulness of proposed distribution in survival and life time analysis. The data set I represents the survival times (in days) of 72 guinea pigs infected with virulent tubercle bacilli, observed and reported by Bjerkedal (1960). The data set I is listed as follows:

$0.1,0.33,0.44,0.56,0.59,0.59,0.72,0.74,0.92,0.93,0.96,1,1,1.02,1.05,1.07,1.07,1.08,1.08$, $1.08,1.09,1.12,1.13,1.15,1.16,1.2,1.21,1.22,1.22,1.24,1.3,1.34,1.36,1.39,1.44,1.46,1.53$, $1.59,1.6,1.63,1.63,1.68,1.71,1.72,1.76,1.83,1.95,1.96,1.97,2.02,2.13,2.15,2.16,2.22,2.3$, $2.31,2.4,2.45,2.51,2.53,2.54,2.54,2.78,2.93,3.27,3.42,3.47,3.61,4.02,4.32,4.58,5.55$.

The data set II represents 84 observations of failure times (in hours) for a particular wind shield model reported by (Murthy et al., 2004):

$0.040,0.301,0.309,0.557,0.943,1.07,1.124,1.248,1.281,1.281,1.303,1.432,1.48,1.505,1.506$, $1.568,1.615,1.619,1.652,1.652,1.757,1.866,1.876,1.899,1.911,1.912,1.914,1.981,2.01,2.038$, $2.085,2.089,2.097,2.135,2.154,2.19,2.194,2.223,2.224,2.229,2.3,2.324,2.385,2.481,2.61$, $2.625,2.632,2.646,2.661,2.688,2.823,2.89,2.902,2.934,2.962,2.964,3,3.103,3.114,3.117$, $3.166,3.344,3.376,3.443,3.467,3.478,3.578,3.595,3.699,3.779,3.924,4.035,4.121,4.167,4.24$, $4.255,4.278,4.305,4.376,4.449,4.485,4.57,4.602,4.663$.

In order to compare the distributions, we consider the following criteria Kolmogorov-Smirnov (K-S) test along with its $p$-value, Cramér-von Mises ( $\left.\mathrm{W}^{*}\right)$ and Anderson-Darling $\left(\mathrm{A}^{*}\right)$ goodness-offit test statistics. However, the model with minimum values for these statistics could be chosen as the best model to fit these data. Table 5 and Table 6 give the MLEs and their SEs for the two data sets. Also, these tables compare the MOIPL model with other important competitive distributions. Table 5 and Table 6 show that MOIPL model gives the lowest values for the K-S, W*, A* statistics and largest 
Table 6: The MLEs, SEs and goodness-of-fit measures of the models for data set II

\begin{tabular}{|c|c|c|c|c|c|c|}
\hline Model & MLE & SE & $P$-value & K-S & $W^{*}$ & $\overline{\overline{A^{*}}}$ \\
\hline \multirow{4}{*}{ MOIPL } & $\hat{\alpha}=4.533$ & 0.745 & \multirow{4}{*}{0.76141} & \multirow{4}{*}{0.23859} & \multirow{4}{*}{0.09219} & \multirow{4}{*}{0.66719} \\
\hline & $\hat{\beta}=0.144$ & 0.136 & & & & \\
\hline & $\hat{\delta}=20.905$ & 39.357 & & & & \\
\hline & $\hat{\theta}=0.043$ & 0.086 & & & & \\
\hline \multirow{4}{*}{ KWL } & $\hat{\alpha}=12.516$ & 16.647 & \multirow{4}{*}{0.74421} & \multirow{4}{*}{0.25579} & \multirow{4}{*}{0.10498} & \multirow{4}{*}{0.95294} \\
\hline & $\hat{\beta}=69.807$ & 88.159 & & & & \\
\hline & $\hat{\lambda}=2.795$ & 0.365 & & & & \\
\hline & $\hat{\theta}=13.393$ & 9.952 & & & & \\
\hline \multirow{3}{*}{ IPL } & $\hat{\alpha}=0.270$ & 0.061 & \multirow{3}{*}{0.73210} & \multirow{3}{*}{0.26790} & \multirow{3}{*}{0.11870} & \multirow{3}{*}{0.98121} \\
\hline & $\hat{\lambda}=0.179^{*}$ & $0.061^{*}$ & & & & \\
\hline & $\hat{\beta}=6.611$ & 0.306 & & & & \\
\hline \multirow{3}{*}{ EL } & $\hat{\lambda}=0.011$ & 0.011 & \multirow{3}{*}{0.16293} & \multirow{3}{*}{0.83707} & \multirow{3}{*}{0.19022} & \multirow{3}{*}{1.58427} \\
\hline & $\hat{\alpha}=3.591$ & 0.623 & & & & \\
\hline & $\hat{\theta}=72.014$ & 72.654 & & & & \\
\hline \multirow{3}{*}{ IEL } & $\hat{\alpha}=150.614$ & 46.402 & \multirow{3}{*}{0.73210} & \multirow{3}{*}{0.26790} & \multirow{3}{*}{2.02712} & \multirow{3}{*}{11.08269} \\
\hline & $\hat{\theta}=1.523$ & 0.316 & & & & \\
\hline & $\hat{\lambda}=0.011$ & $3.761^{*}$ & & & & \\
\hline
\end{tabular}

* Indicate that the value multiply $10^{-3}$

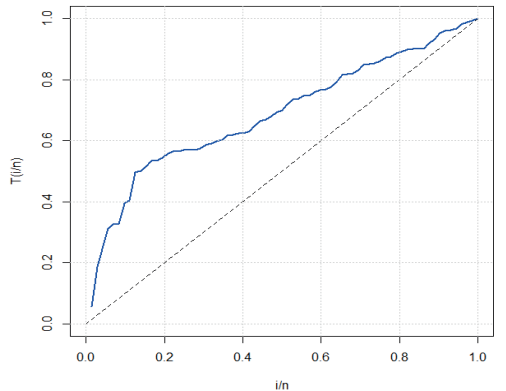

(a)

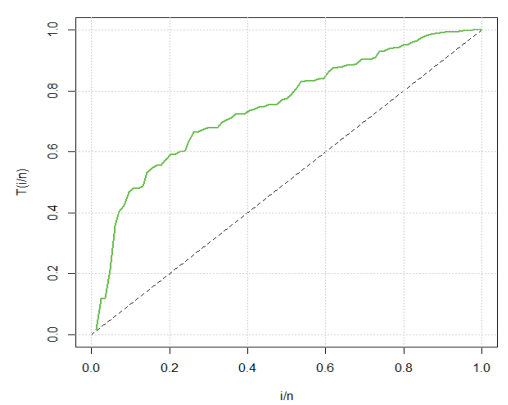

(b)

Figure 12: TTT plots (a) The data set I and (b) The data set II.

p-value among all fitted models.

In the applications, the information about the hazard shape can help in selecting a particular model. For this purpose, we refine our descriptive analysis by showing the corresponding total time test (TTT) plot. Figure 12 presents TTT plots of two data sets, they show a concave curve indicating that an increasing hrf for the fitting model is appropriate, then MOIPL distribution which has increasing shape of hazard function would be appropriate for analyzing these lifetime data.

Furthermore, we plot the histogram, estimated pdf and PP plots for all models for both data. Plots of empirical cdf, estimated pdf, PP plots and QQ plots of MOIPL distribution for both data are displayed (Figure 13 and Figure 14).

Furthermore, the MLEs and BEs of parameters of MOIPL distribution and the corresponding SEs and MC error for both real data sets based on TIC are listed in Table 7.

Based on Table 7, we conclude the following

- It is clear that SEs and MC error of BEs and MLEs decrease as sample size increases.

- The SEs of the MLEs for real data set II get the smallest values compared to the real data set I. 

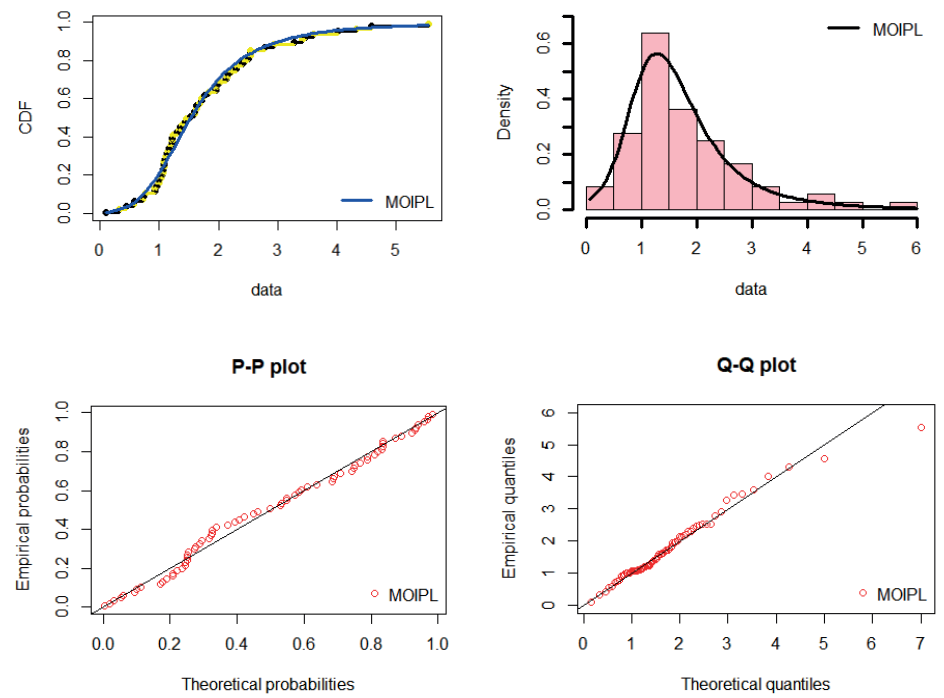

Figure 13: Fitted pdf, cdf, PP-plot and QQ-plot of MOIPL distribution for data set I.
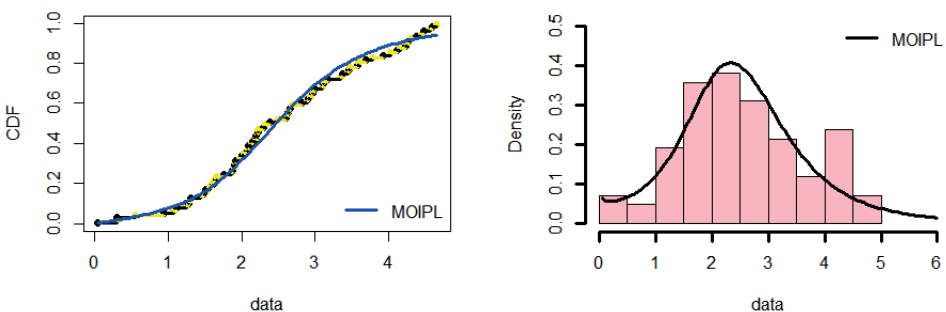

data
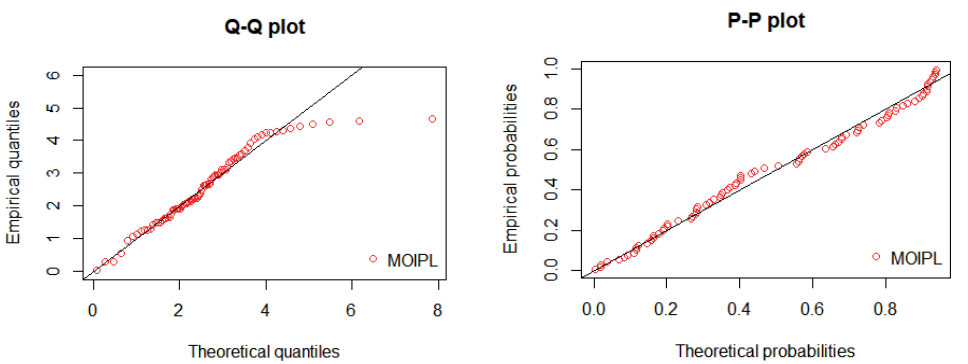

Figure 14: Fitted pdf, cdf, PP-plot and QQ-plot of MOIPL distribution for data set II.

- The SEs of the BEs in the real data set I get the smallest values compared to the real data set II.

- For both real data, the SEs of the parameters $\alpha$ and $\delta$ take the smallest values compared to other parameters based on ML method. While, the SEs of the parameter $\beta$ get the smallest values compared to the other parameters based on Bayesian method.

- Generally speaking, application to the considered real data confirm the above theoretical results. 
Table 7: MLEs, BEs, SEs, and MC error for MOIPL distribution for both data sets

\begin{tabular}{|c|c|c|c|c|c|c|c|}
\hline \multirow{2}{*}{ Real data } & \multirow{2}{*}{$n$} & \multirow{2}{*}{ Parameters } & \multicolumn{2}{|c|}{ ML } & \multicolumn{3}{|c|}{ Bayesian } \\
\hline & & & Estimate & $\mathrm{SE}$ & Estimate & SE & MC error \\
\hline \multirow{12}{*}{ I } & \multirow{4}{*}{30} & $\alpha$ & 1.5223 & 0.3294 & 0.7073 & $5.9120^{*}$ & $0.3387^{*}$ \\
\hline & & $\beta$ & 2.2031 & 0.9603 & 0.2491 & $3.3033 *$ & $0.1958^{*}$ \\
\hline & & $\delta$ & 1.3261 & 0.2170 & 0.4947 & 0.0388 & $3.3730^{*}$ \\
\hline & & $\theta$ & 0.9672 & 0.8822 & 0.1937 & 0.0791 & $7.9430^{*}$ \\
\hline & \multirow{4}{*}{40} & $\alpha$ & 1.5548 & 0.2593 & 0.7083 & $5.7720^{*}$ & $0.3181^{*}$ \\
\hline & & $\beta$ & 2.3689 & 0.9081 & 0.2488 & $3.0040 *$ & $0.1913 *$ \\
\hline & & $\delta$ & 1.3128 & 0.1792 & 0.4940 & 0.0381 & $3.2280^{*}$ \\
\hline & & $\theta$ & 1.4894 & 0.7959 & 0.1690 & 0.0703 & $7.0620 *$ \\
\hline & \multirow{4}{*}{50} & $\alpha$ & 1.6521 & 0.2273 & 0.7076 & $5.7560^{*}$ & $0.3146^{*}$ \\
\hline & & $\beta$ & 2.4021 & 0.8374 & 0.2590 & $2.9750^{*}$ & $0.1883^{*}$ \\
\hline & & $\delta$ & 1.4028 & 0.1611 & 0.4946 & 0.0357 & $3.1288^{*}$ \\
\hline & & $\theta$ & 1.3000 & 0.6130 & 0.1760 & 0.0603 & $7.0570^{*}$ \\
\hline \multirow{12}{*}{ II } & \multirow{4}{*}{30} & $\alpha$ & 1.3521 & 0.2298 & 0.6875 & $5.1320 *$ & $0.2981^{*}$ \\
\hline & & $\beta$ & 1.8764 & 0.7195 & 0.2286 & $2.1190^{*}$ & $0.3894^{*}$ \\
\hline & & $\delta$ & 1.8500 & 0.3417 & 0.9474 & 0.0365 & $3.5670 *$ \\
\hline & & $\theta$ & 1.3552 & 0.9905 & 0.1815 & 0.0676 & $6.7890 *$ \\
\hline & \multirow{4}{*}{40} & $\alpha$ & 1.4269 & 0.2138 & 0.6588 & $5.1020^{*}$ & $0.2874^{*}$ \\
\hline & & $\beta$ & 2.2145 & 0.7091 & 0.2291 & $1.8020^{*}$ & $0.1047 *$ \\
\hline & & $\delta$ & 1.7639 & 0.2237 & 0.9418 & 0.0352 & $3.0610 *$ \\
\hline & & $\theta$ & 1.2670 & 0.7104 & 0.1228 & $7.2720^{*}$ & $0.7245^{*}$ \\
\hline & \multirow{4}{*}{50} & $\alpha$ & 1.5354 & 0.1940 & 0.6485 & $4.7730^{*}$ & $0.2451^{*}$ \\
\hline & & $\beta$ & 2.4250 & 0.6795 & 0.2485 & $1.4515^{*}$ & $0.1016^{*}$ \\
\hline & & $\delta$ & 1.6546 & 01623 & 0.9403 & 0.0254 & $3.0224 *$ \\
\hline & & $\theta$ & 1.0480 & 0.5057 & 0.2031 & $6.5530^{*}$ & $0.6574^{*}$ \\
\hline
\end{tabular}

* Indicate that the value multiply $10^{-3}$

\section{Concluding remarks}

In this paper, we introduce a new four-parameter distribution, called Marshall-Olkin inverse power Lomax distribution, which extends the inverse power Lomax distribution (Hassan and Abd-Alla, 2019). We provide some of its mathematical and statistical properties. We obtain explicit expressions for the ordinary and incomplete moments, negative moments, moments of the residual life. We also derive Rényi entropy and discuss the stochastic ordering. Maximum likelihood and Bayesian methods of estimation are employed to estimate the population parameters based on Type I censored samples. An approximate confidence intervals and Bayesian credible intervals for the population parameters are discussed. Simulation study is provided to assess the model performance of parameters. Two applications illustrate that the suggested distribution provides better fit than other competitive distributi ons.

\section{Acknowledgments}

The authors are grateful to the editor and referees for their useful comments and suggestions for improvement which undoubtedly enhanced the presentation and quality of the article.

\section{References}

Abdul-Moniem IB and Abdel-Hameed HF (2012). A lifetime distribution with decreasing failure rate, International Electronic Journal of Mathematics Education, 33, 1-7.

Al-Marzouk S, Jamal F, Chesneau C, and Elgarhy M (2020). Type II Topp Leone power Lomax 
distribution with applications, Mathematics, 8, 1-26.

Atkinson AB and Harrison A (1978). Distribution of Personal Wealth in Britain, Cambridge University Press, Cambridge.

Bantan R, Hassan AS, and Elsehetry M (2020). Generalized Marshall Olkin Inverse Lindley Distribution with Applications, Computers, Materials, Continua, 64, 1505-1526.

Bjerkedal T (1960). Acquisition of resistance in guinea pigs infected with different doses of virulent tubercle bacilli, American Journal of Hygiene, 72, 130-148.

Cordeiro GM, Ortega E, and Popović B (2013). The gamma Lomax distribution, Journal of Statistical Computation and Simulation, 85, 305-319.

Ghitany ME, AL-Awadhi FA, and Alkhalfan LA (2007). Marshall-Olkin extended Lomax distribution and its applications to censored data, Communications in Statistics: Theory and Methods, 36, 1855-1866.

Haq MA, Hamedani GG, Elgarhy M, and Ramos PL (2020). Marshall-Olkin power Lomax distribution: properties and estimation based on complete and censored samples, International Journal of Statistics and Probability, 9, 48-62.

Harris CM (1968). The Pareto distribution as a queue service discipline, Operational Research, 16, 307-313.

Hassan A, Ascsar S, and Shelbaia A (2016). Optimum step stress accelerated life test plan for Lomax distribution with an adaptive type-II progressive hybrid censoring, British Journal of Mathematics Computer Science, 13, 1-19.

Hassan AS and Abd-Allah M (2017). Exponentiated Lomax geometric distribution: properties and applications, Pakistan Journal of Statistics and Operation Research, 13, 545-566.

Hassan AS and Abd-Allah M (2018). Exponentiated Weibull-Lomax distribution: properties and estimation, Journal of Data Science, 16, 277-298.

Hassan AS and Abd-Allah M (2019). On the inverse power Lomax distribution, Annals of Data Science, 6, 259-278.

Hassan AS and Al-Ghamdi A (2009). Optimum step stress accelerated life testing for Lomax distribut ion, Journal of Applied Sciences Research, 5, 2153-2164.

Hassan AS, Elgarhy M, and Mohamed RE (2020). Statistical properties and estimation of type II half logistic Lomax distribution, Thailand Statistician, 18, 290-305.

Hassan AS and Mohamed RE (2019). Parameter estimation for inverted exponentiated Lomax distribut ion with right censored data, Gazi University Journal of Science, 32, 1370-1386.

Hassan AS and Mohamed RE (2019). Weibull inverse Lomax distribution right censored data, Pakistan Journal of Statistics and Operation Research, 15, 587-603.

Hassan AS and Nassr SG (2018). Power Lomax Poisson distribution: properties and estimation, Journal of Data Science, 18, 105-128.

Hassan AS, Sabry MAS, and Elsehetry A (2020). Truncated power Lomax distribution with application to flood data, Journal of Statistics Applications Probability, 9, 347-359.

Holland O Golaup A, and Aghvami AH (2006). Traffic characteristics of aggregated module downloads for mobile terminal reconfiguration, IEE Proceedings Communications, 153, 683-690.

Krishna E, Jose KK, Alice T, and Ristić MM (2013). The Marshall-Olkin Fréchet distribution, Communications in Statistics: Theory and Methods, 42, 4091-4107.

Lemonte AJ and Cordeiro GM (2013). An extended Lomax distribution, Statistics, 47, 800-816.

Marshall AW and Olkin I (1997). A new method for adding a parameter to a family of distributions with applications to the exponential and Weibull families, Biometrika, 84, 641-52.

M-Gharib, Mohammed BI, and Aghel WER (2017). Marshll-Olkin extended inverse Pareto distribution 
and its application, International Journal of Statistics and Probability, 6, 71-84.

Murthy DNP, Xie M, and Jiang R (2004). Weibull Models, Series in Probability And Statistics, John Wiley, New Jersey.

Pogány TK, Saboor A, and Provost S (2015). The Marshall-Olkin exponential Weibull distribution, Hacettepe Journal of Mathematics and Statistics, 44, 1579-1594.

Rady EA, Hassanein WA, and Elhaddad TA (2016). The power Lomax distribution with an applic ation to bladder cancer data, SpringerPlus, 5, 1838.

Rao GS, Ghitany ME, and Kantam RRL (2009). Marshall-Olkin extended Lomax distribution: an economic reliability test plan, International Journal Of Applied Mathematics, 22, 139-148.

Shaked M and Shanthikumar J (1994). Stochastic Orders and Their Applications, Academic Press, Boston

Tahir M Cordeiro GM Mansoor M, and Zubair M (2015). The Weibull-Lomax distribution: properties and applications, Hacettepe Journal of Mathematics and Statistics, 44, 461-480.

Tahir MH, Hussain MA, Cordeiro GM, Hamedani GG, Mansoor M, and Zubair M (2016). The Gumbel-Lomax distribution: properties and applications, Journal of Statistical Theory and Applic ations, 15, 61-79. 